James A. Charles

Received: 7 January 2007

Accepted in revised form: 1 February 2007

Published online: 11 May 2007

\title{
Early treatment of juvenile migraine reduces disability with disease modification implications
}

Sir,

The prevalence of migraine in children and adolescents under 18 years is rising, and by age $15,75 \%$ of children will experience a significant headache [1]. Although studies show that migraine headaches remit in $17 \%-34 \%$ of adolescent subjects, headaches persist in $20 \%-48 \%$ of subjects, and transform into other types of headaches in $11 \%-37 \%$. In a recent study of 55 subjects aged 11-14 with migraine headaches at baseline, $38.2 \%$ had experienced remission, but $41.8 \%$ had persistent migraine and $20.0 \%$ transformed to tension-type headache [2]. Misunderstanding and inadequate treatment can add to the disabling burden of migraine. This causes loss of school, social and valuable extracurricular participation in a subset of patients. Compounding the problem is the lack of FDA-approved triptan therapies in patients less than 18 years old, in spite of numerous articles demonstrating the effectiveness of triptans [3]. Both central and peripheral components of the trigeminal pain pathway play a significant role in the symptoms and signs of the migraine attack. Triptans are the most effective agents that can suppress peripheral sensitisation [4].

Radiographic, epidemiologic and pathophysiological studies reveal migraine trigeminovascular secondorder neurons and other brainstem generators have become centrally sensitised and refractory in many adults. Welch et al. [5] found that iron deposition occurs in the periaqueductal grey (PAG) in patients with frequent migraines that evolved into chronic migraine. Kruit and colleagues [6] studied 435 adults with migraine \pm aura and controls utilising MRI imaging and found the risk of higher white matter lesion burden on MRI increased with increasing attack frequency. Burstein and Jakubowski demonstrated that central sensitisation explains the progression of attacks, but also may play a role in the progression of the disease itself. Repeated central sensitization episodes are associated with permanent neuronal damage, preventative treatment refractoriness and disease progression [7].

Many adults with chronic daily headache not due to medication overuse did not receive abortive or preventative treatment by a headache expert at onset in childhood and adolescence. Preventative (prophylactic) treatment works best if instituted prior to the development of daily headache patterns [8]. Effective, aggressive and early pharmacological 
treatment of the young migraineur in the early stage of headache presentation will not only reduce headache disability, but may be the venue for migraine disease modification.
James A. Charles, MD,

FAAN, Department of Neurosciences, New Jersey Medical School, 185 S Orange Ave. H-506, Newark, NJ 07103, USA e-mail: jamesacharlesmd@aol.com $\mathrm{Tel}+1-201-858-2457$

Fax $+1-201-858-1053$

\section{References}

1. Bille B (1962) Migraine in school children. A study of the incidence and short-term prognosis, and a clinical, psychological and electroencephalographic comparison between children with migraine and matched controls. Acta Paediatr Suppl 136:1-151

2. Monastero R, Camarda C, Pipia C, Camarda R (2006) Prognosis of migraine headaches in adolescence. Neurology 67:1353-1356

3. Winner P (2002) Triptans for migraine management in adolescents. Headache 42:675-679
4. Buzzi MG, Moskowitz MA (2005) The pathophysiology of migraine: year 2005. J Headache Pain 6:105-111

5. Welch KM, Nagesh V, Aurora SK, Gelman N (2001) Periaqueductal gray matter dysfunction in migraine: cause or the burden of illness? Headache 41:629-37

6. Kruit MC, van Buchem MA, Hofman PA et al (2004) Migraine as a risk factor for subclinical brain lesions. JAMA 291:427-434
7. Burstein R, Jakubowski M (2004) Analgesic triptan action in an animal model of intracranial pain: a race against the development of central sensitization. Ann Neurol 55:27-36

8. Rothrock JF, Kelly NM, Brody ML, Golbeck A (1994) A differential response to treatment with divalproex sodium in patients with intractable headache. Cephalalgia 14:241-244 